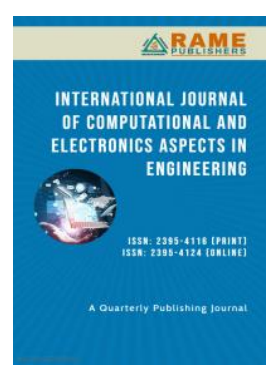

Pwasong A. Davou ${ }^{1}$ davougus@gmail.com

\section{Saratha Sathasivam ${ }^{2}$ saratha@usm.my}

Universiti Sains Malaysia School of Mathematical Sciences, Pulau Pinang, Malaysia

\title{
Statistical Neural Networks in the Classification of Alcoholic Liver Disease and Nonalcoholic Fatty Liver Disease
}

Abstract-This paper deals with the performance of statistical neural network in the classification of alcoholic liver disease (ALD) data and nonalcoholic fatty liver disease data (NAFLD). The study involved 73 individuals that were clinically diagnosed of alcoholic liver disease (ALD) and 80 individuals who were clinically diagnosed of nonalcoholic fatty liver disease (NAFLD). Four different neural network structure, multi-layer perceptron, radial basis function, probabilistic neural network and generalized regression neural network were applied to the data to determine the performance of statistical neural networks in the classification of liver disease data. The overall result indicates that the most suitable statistical neural network model for classifying ALD and NAFLD data is the probabilistic neural network (PNN) with a $95.7 \%$ classification performance and 67 correct classifications. Radial basis function network (RBF) and multilayer perceptron network (MLP) has the lowest classification accuracy with 55 classified samples each. The generalized regression neural network (GRNN) was the second-best network with 62 correct classifications. The computer simulation was carried out by using MATLAB 6.0 Neural Network Toolbox.

Index Terms - Classification, neural network, probabilistic neural network, liver and diseases

\section{INTRODUCTION}

In an assertion by Ugiagbe [1] shows that the liver is a vital organ located in the upper right-hand side of the abdomen. It is as big as a football, weights about 1kilogram 360.78grams, and performs many functions for the body. The tasks of liver in the body comprises: digesting and purifying elements that would otherwise be harmful to the body, transforming nutrients derived from

Technical Article

First Online on - 30 March 2015, Revised on - 30 June 2020

(C) 2020 RAME Publishers

This is an open access article under the CC BY 4.0 International License https://creativecommons.org/licenses/by/4.0/

Cite this article - Pwasong A. Davou and Saratha Sathasivam, "Statistical Neural Networks in the Classification of Alcoholic Liver Disease and Nonalcoholic Fatty Liver Disease", International Journal of Computational and Electronics Aspects in Engineering, RAME Publishers, vol. 1, issue 2, pp. 56-62, 2015, Revised in 2020.

https://doi.org/10.26706/ijceae.1.2.20150102 food into vital blood constituents, modifying blood thickening, manufacturing proteins and enzymes, upholding hormone balances, and stowing various vitamins. The liver also brands features that help the human immune system combat contamination, eradicates bacteria from the blood, and brands bile, which is essential for digestion. Liver disease is the overall word used for any sickness of the liver. Some reasons of liver disease can include injury, impurity, or a hereditary defect. Some of the causes of liver disease can translate to hepatitis.

Vanni et al. [2] has defined fatty liver as the abnormal accumulation of fats inside the liver cells and usually occurs in people who are obese (overweight), diabetic (high blood sugar) or have elevated cholesterol or triglyceride (high levels of fats in the

blood) in their blood. It can also occur with unwarranted or protracted alcohol drinking. It is further 
stated in [2] that fatty liver often does not cause symptoms but sometimes a person with fatty liver may feel tired or have vague abdominal discomfort. If the reason of fatty liver is not determined, the liver may turn out to be swollen (hepatitis) and cause a further grave problem.

Alcoholic liver disease is defined as injury to the liver and its function due to alcohol abuse. Alcoholic liver sickness occurs after years of heavy drinking. Alcohol can cause inflammation in the liver. Over time, disfiguring and cirrhosis can occur. Cirrhosis is the final phase of alcoholic liver disease. They further reported that alcoholic liver disease does not occur in all heavyweight drinkers. The chances of getting liver disease go up the longer you have been drinking and the more alcohol you consume. You do not have to get drunk for the disease to occur. The disease seems to be more common in some families. Women may be more likely to have this problem than men. This was reported by O'Shea et al. [3].

A neural network is also acknowledged as artificial neural network (ANN), connectionist model or parallel distributed treating model. Neural networks are immensely parallel interrelated networks of simple elements and their classified organizations which are proposed to interact with objects of the physical world in the same way as biological nervous systems do. It is an information handling pattern that is inspired by the way the brain processes information at the low biological level. It look like the brain in terms of knowledge being developed by the network through a learning process and inter-neuron linking strengths recognized as synaptic weights which are used to store the knowledge. These are extracts from Rajakarunakaran et al. [4].

Pye and Bangham [5] assert that when it is determined that an object from a population $\mathrm{p}$ belongs to a known subpopulation s, it is said that pattern recognition is done. He further stated that the recognition of an individual object as a unique singleton class is called identification. Classification is defined by Meech et al. [6] as the process of grouping objects together into classes (subpopulations) according to their perceived likeness. The subject area of pattern recognition includes both classification and recognition and belongs to the broader field of machine intelligence - that is, the study of how to make machines learn and reason to make decisions, as do humans. In an assertion by Sivanandam and Deepa [7] shows that classification process in neural network consists of three major phases- recognition layer in which the pattern to be classified is applied for each neuron in the recognition layer, the comparison phase is a layer in which the single network firing in the recognition layer passed a 1 back to the comparison layer on its output signal and the third phase called the search layer whereby if there is no reset signal generated, the match is adequate and the classification is finished. Otherwise, the other stored pattern must be researched to seek a correct matched.

$\mathrm{Wu}$ et al. [8] engaged probabilistic neural network (PNN) with image and data processing methods to device general purpose computerized leaf recognition for plant classification. The PNN was trained by 1800 leaves to classify 32 kinds of plants with precision superior than $90 \%$. Compared with other approaches; their algorithm is a precise artificial intelligent approach which is fast in performance and easy in enactment.

This study applied radial basis function (RBF) network, generalized regression neural network (GRNN) and probabilistic neural network (PNN) to classify alcoholic liver disease data and nonalcoholic liver disease data. The study also used results obtain to compare with multilayer perceptron (MLP) network.

This paper is organized as follows. In section 2 the methods of statistical neural networks are describe. Simulations results of data on alcoholic liver disease and nonalcoholic fatty liver disease are presented in section 3 . The conclusion and future work are given in section 4 .

\section{Methods Of Statistical NeURAL NeTwORKS}

\section{A. Radial Basis Functions (RBF)}

A radial basis function ( $\mathrm{RBF}$ ) network is typically a three-layer network, containing of an input layer, a hidden layer, and an output layer. The network fascinates a great 
deal of interest due to fast training and simplicity. The hidden layer of the RBF network comprises of many neurons that form a parameter vector called - center. The center can be measured as the weight vector of the hidden layer. A distance is used to quantify how far an input vector is from the center. Hidden neurons use Gaussian functions as activation functions as asserted by Kiyan and Yildirim [9].

RBF is a different approach by viewing the design of a neural network as a curve-fitting problem in a highdimensional space. According to this perspective, learning is the same as finding a surface in a multidimensional space that affords a best fit to the training data, with the criterion for "best fit" being measured in some statistical sense. The building of a radial-basis function network in its greatest basic form comprises three entirely unlike layers. The input layer is made up of source nodes. The second layer is a hidden layer of sufficient dimension, which serves a different resolution from that in a multilayer perceptron. The output layer supplies the reaction of the network to the activation patterns applied to the input layer. The change from the input space to the hidden-unit space is nonlinear while the change from the hidden-unit space to the output space is linear.

\section{B. Probabilistic Neural Networks (PNN)}

The probabilistic neural network (PNN) presented by Specht is fundamentally based on the well-known Bayesian classifier technique commonly used in numerous classical pattern-recognition problems. Consider a pattern vector $\mathrm{x}$ with $\mathrm{m}$ dimensions that fits to one of two categories $\mathrm{V}_{1}$ and $\mathrm{V}_{2}$. Let $\mathrm{R}_{1}(\mathrm{x})$ and $\mathrm{R}_{2}(\mathrm{x})$ be the probability density functions (pdf) for the classification categories $V_{1}$ and $V_{2}$, respectively. From Bayes' discriminant decision rule, $\mathrm{x}$ belongs to $\mathrm{V}_{1}$ if

$$
\frac{R_{1}(x)}{R_{2}(x)}>\frac{L_{1}}{L_{2}} \frac{P_{2}}{P_{1}}
$$

Conversely, $\mathrm{x}$ belongs to $\mathrm{V}_{2}$ if

$$
\frac{R_{1(x)}}{R_{2(x)}}<\frac{L_{1}}{L_{2}} \frac{P_{2}}{P_{1}}
$$

where $L_{1}$ is the loss or cost function associated with misclassifying the vector as belonging to category $\mathrm{V}_{1}$ while it belongs to category $\mathrm{V}_{2}, \mathrm{~L}_{2}$ is the loss function associated with misclassifying the vector as belonging to category $\mathrm{V}_{2}$ while it belongs to category $\mathrm{V}_{1}, \mathrm{P}_{1}$ is the prior probability of occurrence $\mathrm{V}_{1}$, and $\mathrm{P}_{2}$ is the prior probability of occurrence of category $\mathrm{V}_{2}$. In many situations, the loss functions and the prior probabilities can be considered equal. Hence the key to using the decision rules given by equations (1) and (2) is to estimate the probability density functions from the training patterns. This was reported by kubat [10].

In the PNN, a nonparametric estimation technique known as Parzen windows is used to construct the classdependent probability density functions (pdf) for each classification category required by Bayes' theory. This allows determination of the chance a given vector pattern lies within a given category. Combining this with the relative frequency of each category, the PNN selects the most likely category for the given pattern vector. Both Bayes' theory and Parzen windows are theoretically well established, have been in use for decades in many engineering applications, and are treated at length in a variety of statistical textbooks. If the jth training pattern for category $V_{1}$ is $x_{j}$, then the Parzen estimate of the pdf for category $V_{1}$ is

$R_{1}(x)=\frac{1}{(2 \pi)^{m / 2 \sigma^{m} n}} \sum \exp \left[-\frac{\left(x-x_{j}\right)^{\tau}\left(x-x_{j}\right)}{2 \sigma^{2}}\right]$

where $\mathrm{n}$ is the number of training patterns, $\mathrm{m}$ is the input space dimension, $\mathrm{j}$ is the pattern number, and $\sigma$ is an adjustable smoothing parameter. However, the choice of $\sigma$ in general has been found to be not too sensitive to variations in its value.

Specht [11] also asserts that probabilistic neural networks can be used for classification problems. Once an input is accessible, the first layer calculates distances from the input vector to the training input vectors and creates a vector whose components designate how handy the input is to a training input. The second layer adds these contributions for every class of inputs to create as its net 
output a vector of probabilities. Finally, a broad transfer function on the output of the second layer picks the extreme of these probabilities, and produces a 1 for that class and a 0 for the other classes.

\section{Generalized Regression Neural Networks (GRNN)}

The generalized regression neural networks (GRNNs) are the archetypes of radial basis function (RBF) networks, frequently used for function estimates. It's alternative term for Nadaraya Watson kernel regression, and has the following form for the function mapping.

$$
W(x)=\frac{\sum_{k} t_{k} \exp \left\{-|| x-x_{j} \|^{2} / 2 h^{2}\right\}}{\sum_{k} \exp \left\{-|| x-x_{k} \|^{2} / 2 h^{2}\right\}}
$$

Setiano [12] assert that GRNNs share a distinct property, specifically that they do not require iterative training; the hidden to-output weights are just the target values $t_{k}$, so the output $\mathrm{W}(\mathrm{x})$, is merely a weighted average of the target values $t$ of training cases $x$ neighboring to the given input case $\mathrm{x}$. It can be regarded as a standardized RBF network in which there is a hidden unit centered at each training instance. These RBF units are called "kernels" and are typically probability density functions such as the Gaussians reflected in (4). The only weight that requires to be learned is the width of the RBF units $h$. These widths (often a single width is used) are termed "smoothing parameters" or "bandwidths" and are usually chosen by cross validation. GRNN is a universal estimator for smooth functions, so it should be able to solve every smooth function estimation problem given enough data. The foremost disadvantage of GRNNs is that, like kernel approaches in general, they suffer earnestly from the obscenity of dimensionality. GRNNs cannot ignore irrelevant inputs without major modifications to the basic algorithm.

\section{DisCUSSION OF SIMULATION RESULTS}

In this study a random sample of 200 alcohol consumers was randomly selected from a population of 500 alcohol consumers in a particular local population and tested medically to determine their alcoholic liver disease status. Out of the 200 tested, 73 were diagnosed of the disease. This group will be term the first group and will constitute the alcoholic liver disease (ALD) category. The second group will constitute the nonalcoholic fatty liver disease category. The data for the second group was obtained from a random sample of 200 obese and diabetic patients attending a clinic was randomly selected from a population of 500 and tested medically to determine their nonalcoholic fatty liver (NAFLD) disease status. Out of the 200 tested, 80 were diagnosed of the disease. There are two values in the class variable of liver disease: Alcoholic liver disease (ALD) and nonalcoholic fatty liver disease (NAFLD), which is symbolized numerically by 1 and -1 respectively. Table I shows the data distribution.

TABLE I.

Classes AND THEIR DATA DisTRIBUTIONS

\begin{tabular}{|c|c|c|c|}
\hline Class & Total & No. of training data & No. of test data \\
\hline 1 & 73 & 40 & 33 \\
\hline-1 & 80 & 43 & 37 \\
\hline
\end{tabular}

The simulations were realized by using MATLAB 6.0 Neural Network Toolbox. Four different neural network structure, multi-layer perceptron, radial basis function, probabilistic neural network and generalized regression neural network were applied to the liver disease data to show the performance of statistical neural networks on alcoholic liver disease (ALD) and nonalcoholic fatty liver disease (NAFLD) data. The spread value of RBF, PNN and GRNN was chosen as 3.4, 1.5 and 2.5, respectively. In MLP, learning rate was 0.6 .

The data that was used for the training include 40 samples of the training data which belong to the ALD class and 43 samples belong to the NAFLD class. The classification results of the training set by GRNN, RBF, PNN and MLP are presented in tables II, III, IV, and V.

TABLE II.

Classification Of Training Data By RBF

\begin{tabular}{|c|c|c|}
\hline Class & ALD & NAFLD \\
\hline Correct & 40 & 43 \\
\hline Incorrect & 0 & 0 \\
\hline
\end{tabular}

Table II above indicates that 40 of the sample observations in the alcoholic liver disease (ALD) category 
were classified correctly and in the nonalcoholic fatty liver disease (NAFLD) class 43 of the sample observations were classified correctly. None of the sample observations in the ALD and NAFLD categories was classified incorrectly. Hence, the ALD and NAFLD classes had $100 \%$ correct classification in table II as far as classification of the training data by RBF is concern.

TABLE III.

ClassificAtion Of TRAining DATA By PNN

\begin{tabular}{|c|c|c|}
\hline Class & ALD & NAFLD \\
\hline Correct & 40 & 43 \\
\hline Incorrect & 0 & 0 \\
\hline
\end{tabular}

In table III above, classification of the training data by probabilistic neural network (PNN) revealed that both alcoholic liver disease (ALD) category and nonalcoholic liver disease (NAFLD) category were classified correctly, void of any incorrect classification with a classification performance of $100 \%$.

TABLE IV.

CLASSIFICATION OF TRAINING DATA BY GRNN

\begin{tabular}{|c|c|c|}
\hline Class & ALD & NAFLD \\
\hline Correct & 30 & 28 \\
\hline Incorrect & 10 & 15 \\
\hline
\end{tabular}

The classification of the training data by generalized regression network (GRNN) in table IV above showed that 30 of the sample observations in the alcoholic liver disease (ALD) category were classified correctly, while 10 of the remaining sample observations in the ALD class were incorrectly classified. In the nonalcoholic fatty liver disease (NAFLD) category, 28 of the sample observations were classified correctly, while the remaining 15 observations were incorrectly classified in the NAFLD class. The classification results give the performance of the GRNN on the training data to be $69.9 \%$.

TABLE V.

Classification Of Training Data By MLP

\begin{tabular}{|c|c|c|}
\hline Class & ALD & NAFLD \\
\hline Correct & 33 & 35 \\
\hline Incorrect & 7 & 8 \\
\hline
\end{tabular}

The multilayer perceptron was used in the classification of the training data and results are given in table V. The results indicate that 33 of the sample observations in the alcoholic liver disease (ALD) category were correctly classified and 7 sample observations were incorrectly classified. The MLP was also applied in the classification of the training data for the nonalcoholic fatty liver disease (NAFLD) category which revealed that 35 of the sample observations were classified correctly and 8 of the sample observations were classified incorrectly. The performance of the MLP on the training data is $81.9 \%$.

\section{TABLE VI.}

PERFormance Of Training DATA ClASSIFICATION

\begin{tabular}{|c|c|}
\hline Method & Performance \\
\hline RBF & $100 \%$ \\
\hline PNN & $100 \%$ \\
\hline GRNN & $69.9 \%$ \\
\hline MLP & $81.9 \%$ \\
\hline
\end{tabular}

In table VI above, the summary of the performance of the neural networks' RBF, PNN, GRNN and MLP on the training data indicates that RBF and PNN had the best classification performance of $100 \%$ each. The $100 \%$ classification performance produces the best classification accuracy with 82 correct classifications. The GRNN has the least classification performance of $69.9 \%$ on the training data which produces the lowest accuracy with 58 correct classifications for the training set. The MLP classification performance of $81.9 \%$ is next to the performance of the RBF and PNN with 68 correct classifications.

In order to determine the overall performance of the neural network methods considered above for the classification of the training data for alcoholic liver disease (ALD) category and nonalcoholic fatty liver disease (NAFLD) category. The result obtained in tables II, III, IV, $\mathrm{V}$ and VI was considered with the results obtained in the classification of test data by RBF, PNN, GRNN and MLP respectively. Tables VII, VIII, IX and $\mathrm{X}$ indicate the results obtained for the classification of test data by RBF, PNN, GRNN and MLP respectively. Table XI indicates 
the performance of the classification of test data by the four different neural network methods stated above in percentages.

TABLE VII.

Classification Of TeSt DATA By RBF

\begin{tabular}{|c|c|c|}
\hline Class & ALD & NAFLD \\
\hline Correct & 26 & 29 \\
\hline Incorrect & 7 & 8 \\
\hline
\end{tabular}

Table VII above indicates that 26 of the sample observations in the alcoholic liver disease (ALD) category were classified correctly and in the nonalcoholic fatty liver disease (NAFLD) class 29 of the sample observations were classified correctly for test data. 7 of the remaining sample observations in the ALD class were incorrectly classified. In the nonalcoholic fatty liver disease (NAFLD) category for test data, 8 of the sample observations were classified incorrectly. The classification results give the performance of the radial basis function network $(\mathrm{RBF})$ on the test data to be $78.6 \%$.

TABLE VIII.

Classification Of TEST DATA By PNN

\begin{tabular}{|c|c|c|}
\hline Class & ALD & NAFLD \\
\hline Correct & 31 & 36 \\
\hline Incorrect & 2 & 1 \\
\hline
\end{tabular}

Table VIII above indicates that 31 of the sample observations for test data in the alcoholic liver disease (ALD) category were classified correctly and in the nonalcoholic fatty liver disease (NAFLD) class, 36 of the sample observations were classified correctly. 2 of the remaining sample observations in the ALD class were incorrectly classified. In the nonalcoholic fatty liver disease (NAFLD) category for test data, 1 of the sample observation was classified incorrectly. The classification results give the performance of the probabilistic neural network (PNN) on the test data to be $95.7 \%$.

TABLE IX.

ClassificATION OF TEST DATA By GRNN

\begin{tabular}{|c|c|c|}
\hline Class & ALD & NAFLD \\
\hline Correct & 28 & 34 \\
\hline Incorrect & 5 & 3 \\
\hline
\end{tabular}

The classification of the test data by generalized regression network (GRNN) in table IX above showed that 28 of the sample observations in the alcoholic liver disease (ALD) category were classified correctly, while 5 of the remaining sample observations in the ALD class were incorrectly classified. In the nonalcoholic fatty liver disease (NAFLD) category, 34 of the sample observations were classified correctly, while the remaining 3 observations were incorrectly classified in the NAFLD class. The classification results give the performance of the GRNN on the test data to be $88.6 \%$.

TABLE X.

Classification Of Test Data By Mlp (Average)

\begin{tabular}{|c|c|c|}
\hline Class & ALD & NAFLD \\
\hline Correct & 24 & 31 \\
\hline Incorrect & 9 & 6 \\
\hline
\end{tabular}

The multilayer perception was also used in the classification of the test data and results are given in table $X$. The results indicate that 24 of the sample observations in the alcoholic liver disease (ALD) category were correctly classified and 9 sample observations were incorrectly classified. The MLP was also applied in the classification of the test data for the nonalcoholic fatty liver disease (NAFLD) category which revealed that 35 of the sample observations were classified correctly and 8 of the sample observations were classified incorrectly. The performance of the MLP on the test data is $78.6 \%$.

TABLE XI.

Performance Of Test Data By Classification

\begin{tabular}{|c|c|}
\hline Type & Performance \\
\hline RBF & $78.6 \%$ \\
\hline PNN & $95.7 \%$ \\
\hline GRNN & $88.6 \%$ \\
\hline MLP & $78.6 \%$ \\
\hline
\end{tabular}

In table XI above, the summary of the performance of the neural networks' RBF, PNN, GRNN and MLP on the test data indicates that PNN and GRNN had the best classification performance of $95.7 \%$ and $88.6 \%$ respectively. This analogy on the test data produces the 
best classification accuracy for PNN and GRNN with 67 and 62 correct classifications respectively for the test data set. The RBF and MLP have the least classification performance of $78.6 \%$ each respectively. This analogy on the test data respectively produces the lowest accuracy for RBF and MLP with 55 correct classifications each for the test data set.

A total of 70 samples were applied to the networks as test data; that is, $46 \%$ of the data was used for testing. 33 sample observations belong to the ALD class and 37 sample observations belong to the NAFLD class, were chosen for the test. PNN gives the best classification accuracy when the test set is considered. According to the overall results, it is seen that the most suitable neural network model for classifying alcoholic liver disease and nonalcoholic fatty liver disease data is PNN. This work also indicates that statistical neural networks can be effectively used for alcoholic liver disease and nonalcoholic fatty liver disease diagnosis to help hepatologists and gastroenterologists.

\section{CONCLUSIONS}

This study illustrated the manner statistical neural networks are used in actual clinical diagnosis of alcoholic liver disease and nonalcoholic fatty liver disease. By applying statistical neural networks, a diagnostic classification system that performs at an accuracy level is constructed here.

In this work, the performance of statistical neural network structures was investigated for alcoholic liver disease and nonalcoholic fatty liver disease diagnosis problem. RBF and PNN are the best classifiers in the training set, while PNN and GRNN give the best performance for the test data set. In future work, the number of data set may be increase in order to improve the accuracy of the classification performance on each category of liver disease considered in this paper quantitatively.

\section{ACKNOWLEDGMENT}

This research was supported by FRGS grant (203/PMATHS/6711368) offered by Ministry of Higher Education and Universiti Sains Malaysia.

\section{REFERENCES}

[1] Ugiagbe, E. E., \& Udoh, M. O., "The histopathological pattern of liver biopsies at the University of Benin Teaching Hospital", Nigerian journal of clinical practice, 16(4), 2013.

[2] Vanni, E., Bugianesi, E., Kotronen, A., De Minicis, S., YkiJärvinen, H., \& Svegliati-Baroni, G., "From the metabolic syndrome to NAFLD or $<\mathrm{i}>$ vice versa $</ \mathrm{i}>$ ", Digestive and liver Disease, 42(5), 320-330, 2010.

[3] O'Shea, R. S., Dasarathy, S., \& McCullough, A. J. "Alcoholic liver disease". Hepatology, 51(1), 307-328, 2010.

[4] Rajakarunakaran, S., Venkumar, P., Devaraj, D., \& Rao, K. "Artificial neural network approach for fault detection in rotary system". Applied Soft Computing, 8(1), 740-748, 2008.

[5] Pye, C. J., \& Bangham, J. A. "Using a genetic algorithm to adapt $1 \mathrm{~d}$ nonlinear matched sieves for pattern classification in images". In IS\&T/SPIE's Symposium on Electronic Imaging: Science \& Technology (pp. 48-55), 1995. International Society for Optics and Photonics.

[6] Meech, J. A., Kawazoe, Y., Kumar, V., \& Maguire, J. F. (Eds.). Intelligence in a Small Materials World. DEStech Publications, Inc, 2005.

[7] Sivanandam, S. N., \& Deepa, S. N. Introduction to neural networks using Matlab 6.0. Tata McGraw-Hill Education, 2006.

[8] Wu, S. G., Bao, F. S., Xu, E. Y., Wang, Y. X., Chang, Y. F., \& Xiang, Q. L., "A leaf recognition algorithm for plant classification using probabilistic neural network", In Signal Processing and Information Technology, 2007 IEEE International Symposium on (pp. 11-16), 2007. IEEE.

[9] Kiyan, T., \& Yildirim, T. "Breast cancer diagnosis using statistical neural networks", IU-Journal of Electrical \& Electronics Engineering, 4(2), 1149-1153, 2011.

[10] Kubat, M., "Neural networks: a comprehensive foundation by Simon Haykin", Macmillan, 1994, ISBN 0-02-3527817, 1999.

[11] Specht, D. F. Probabilistic neural networks. Neural networks, 3(1), 109-118, 1990.

[12] Setiono, R. "Generating concise and accurate classification rules for breast cancer diagnosis". Artificial Intelligence in medicine, 18(3),205-219, 2000. 